\title{
CIRSE Standards of Practice on Conducting Meetings on Morbidity and Mortality
}

\author{
Joo-Young Chun ${ }^{1}$ (D) Arindam Bharadwaz ${ }^{2} \cdot$ Jimmy Kyaw Tun $^{3} \cdot$ Tiago Bilhim $^{4,5}$. \\ Carla Gonzalez-Junyent ${ }^{6} \cdot$ Bhavin Kawa $^{1}$
}

Received: 17 November 2020/ Accepted: 26 April 2021

(C) Springer Science+Business Media, LLC, part of Springer Nature and the Cardiovascular and Interventional Radiological Society of Europe (CIRSE) 2021

\begin{abstract}
This CIRSE Standards of Practice document is developed by an expert writing group under the guidance of the CIRSE Standards of Practice Committee. It aims to assist Interventional Radiologists in their daily practice by providing best practices for conducting meetings on morbidity and mortality.
\end{abstract}

Keywords Adverse events - Complications . Morbidity and mortality - Systematic analysis . Learning from mistakes $\cdot$ Patient safety

\section{Introduction}

Adverse events are inevitable in medicine across all specialties. The need to learn from adverse outcomes is an essential part of safe clinical practice, especially in specialties centred around performing invasive procedures [1]. Interventional Radiology (IR) is a specialty that offers a wide range of minimally invasive, image-guided treatments

Joo-Young Chun

jooyoung.chun@stgeorges.nhs.uk

1 Department of Radiology, St George's Hospital, London, UK

2 Department of Radiology, Aarhus University Hospital, Aarhus, Denmark

3 Department of Interventional Radiology, Barts Health NHS Trust, London, UK

4 Interventional Radiology Unit, Centro Hospitalar Universitário de Lisboa Central, Saint Louis and CUF Hospital, Lisbon, Portugal

5 Nova Medical School, Lisbon, Portugal

6 Hospital Universitari Vall d'Hebron, Barcelona, Spain for an increasing number of disease processes. The benefits of IR techniques have been recognised by healthcare providers and policy makers, resulting in continued expansion of the scope and complexity of procedures. Interventional Radiologists require specialist training to equip them with the technical skills to perform procedures safely and to a high standard, and the clinical expertise to make appropriate decisions and manage potential complications $[2,3]$.

The need to report and investigate medical adverse events is well recognised. Mortality and morbidity (M\&M) conferences are widely used platforms for clinicians to learn from such events. The origin of the M\&M conference is often attributed to the work of Ernest Armoury Codman, a surgeon at Massachusetts General Hospital who in 1916 proposed a standardised approach to recording outcomes of surgery, including failures and complications, as a means to improve surgical practice $[4,5]$. He categorised adverse outcomes according to factors related to surgeon, disease or patient and considered interventions to prevent similar events from occurring in the future.

The principles underlying the modern-day M\&M conference have evolved mainly through experience in surgery and anaesthesia [5-7]. There is now greater understanding of human factors and errors in medicine, in that the root cause is often not only attributable to an individual, but a result of organisation and system failures [8]. The M\&M conference can be a forum for identifying such issues to provide a basis to implement change and improve patient safety. Contemporary M\&M meetings form an important part of the organisation's governance framework that supports individual learning and encourages examination of current systems and processes [6, 7, 9-13].

There are several studies that support the effectiveness of $\mathrm{M} \& \mathrm{M}$ conferences in reducing mortality. One academic 
surgical unit reported $40 \%$ reduction in surgical mortality four years after introducing robust elements to M\&M conferences [14], and another large surgical centre reported significant reduction in observed-to-expected mortality ratios from 1.14 to 0.75 one year after introduction of a standardised M\&M review process [15]. Recent public enquiries into poor post-operative outcomes have also emphasised the importance of M\&M meetings [16, 17]. Indeed, attendance at M\&M meetings is mandatory for surgeons in the United States [18, 19], and all surgeons in the United Kingdom are also expected to attend according to best practice guidelines [7]. Despite the growing evidence and the general consensus that M\&M meetings are useful and a necessary part of good clinical practice, there is wide variation in the format and level of participation $[9,12,20]$. There is also limited guidance on how they should be conducted in order to maximise benefit $[1,10,12,13]$.

There are known barriers to effective M\&M meetings. These include unsupportive organisational and professional culture, lack of education regarding the process, anxiety in exposing individual fault, fear of legal ramifications and lack of time $[6,12,21]$. Historically, some M\&M conferences had a 'blame and shame' culture, which is not conducive to openness and transparency [22]. The nature of learning from mistakes is a delicate issue and it is important that M\&M meetings facilitate open discussion, sharing of experiences and balanced examination of difficult cases[6].

There is little in the literature with regard to experience of M\&M meetings in IR, as is the case in most other specialties outside of surgery and anaesthesia. Of note, one academic paediatric IR unit reported a large retrospective review of 10 years' experience of regular M\&M meetings and concluded that they provide a useful forum for team discussion and potential for quality improvement [9]. The authors reflected that M\&M discussions allow implementation of simple and practical changes with buy-in from team members. Sharing tips and solutions to problems also promoted more uniform practice, standardisation of procedures and therefore greater efficiency.

As our specialty has evolved to encompass increasingly complex procedures in higher-risk patient groups, the potential for complications is rising [23]. Moreover, as IR has always been at the forefront of technological innovation with numerous new devices emerging each year, this creates further possibility for complications related to their use. A survey of British Society of Interventional Radiology (BSIR) members in 2013 revealed that $80 \%$ of respondents experienced an adverse incident related to unexpected failure of a medical device in their IR practice [24]. Medical device failures can cause significant harm to patients and there is increasing public awareness, owing to recent high-profile cases involving surgical breast and hip implants. A UK government review in 2012 emphasised the importance of healthcare professionals to be involved in medical device vigilance by reporting device-related adverse incidents to the Medicines and Healthcare products Regulatory Agency (MHRA)[25]. Similarly, the European Union Medical Devices Directive also highlights the need to improve reporting of device-related incidents in member states to enhance patient safety [26].

The purpose of this document is to provide a framework for conducting M\&M conferences in IR. The aim is to raise awareness and increase implementation of M\&M meetings across the global IR community. The recommendations draw on the existing evidence on how best to conduct $M \& M$ meetings to maximise effectiveness, and incorporates the CIRSE Classification System for Complications [3].

\section{Patient Safety in IR}

There has been a drive in the IR community in recent years to shift focus to the clinical elements of IR practice [2, 27, 28], which emphasise the importance of being a clinician who accepts responsibility for the direct care of patients before and after the procedure. A key facet of a successful clinical IR practice is a governance framework where adverse outcomes are examined and underlying issues are addressed in a timely manner. The commitment to improve and maintain patient safety in IR is outlined by existing CIRSE and SIR Standards of Practice documents, which have been widely adopted. Of note, the CIRSE IR Patient Safety Checklist was published in 2012, adapted from the World Health Organization (WHO) surgical safety checklist, in order to meet the requirements for an IR setting [29]. The use of safety checklists has been associated with a reduction in in-hospital morbidity and mortality, one study reporting reduction in mortality from $1.5 \%$ to $0.8 \%$ and morbidity from 11 to $7 \%$ before and after the introduction of the WHO checklist [30].

More recently, the CIRSE Standards for Classification of Complications were created to address the lack of standardisation of reporting and grading complications related to IR procedures. This classification system combines clinical outcome with severity of sequelae. The simple broad-based criteria can be applied in a uniform way to a wide range of clinical settings and a growing spectrum of IR procedures [3]. The clear definition of each grade aims to minimise potential discrepancies between assessors that have been shown in other classification systems [31].

The concept of discussing and learning from complications in IR has been actively promoted in recent years. 
The International Conference on Complications in IR (ICCIR) meeting is entirely dedicated to this purpose and has been lauded for facilitating open exchange with fellow colleagues about difficult real-life encounters. Many national and international IR congresses have incorporated $M \& M$ case discussion sessions, which are valuable in introducing IRs to the principles and benefits of M\&M meetings. They also provide an opportunity to observe the skills necessary to scrutinise adverse events in a discrete and professional manner.

Although M\&M meetings are a familiar concept among IRs, their adoption into daily clinical practice remains inconsistent [9]. A survey of 150 CIRSE members in 2012 showed that less than $50 \%$ of respondents held departmental M\&M meetings [1]. Unsurprisingly, there was wide variation in the meeting frequency, scope of attendees, case selection and perceived value. Although the majority of IRs reported that $M \& M$ meetings are beneficial to their practice and others would be interested in incorporating these meetings, they are not routinely implemented in many IR units. The main barriers to not holding such meetings were reported to be lack of time and small size of practice groups. The absence of guidelines and paucity of literature on M\&M meetings in the IR setting also add to the reasons for limited uptake and lack of uniformity in the structure and content of these meetings.

\section{Goals of M\&M Meetings}

M\&M meetings in the modern healthcare setting have a dual purpose. They provide an opportunity for individuals and teams to reflect and learn from events that have led to adverse patient outcomes. They are also a platform to identify and institute change to systems and processes and thereby drive quality improvement and overall service delivery $[1,7,9-13,19,20]$. There has been mixed success in achieving these aims due to the wide variation in the quality of these meetings and the perceived effectiveness of M\&M meetings in improving patient outcome $[6,11,19]$. These have been largely attributed to poorly standardised approaches and variable attendance by key staff members. Given the important role of M\&M meetings in continuing professional development and service improvement, there is now a growing body of evidence around best practice models for conducting M\&M meetings. The factors that maximise the effectiveness of $M \& M$ conferences are explored and form the basis of our recommendations in the following section.

\section{General Guiding Principles}

M\&M meetings should facilitate open discussion that allows objective and non-judgemental examination of adverse events. They are a forum for doctors and the wider multidisciplinary team to address errors and mistakes without fear of retribution or blame [6, 32, 33]. The discussion is therefore focussed on systems and processes rather than individual fault [13]. It is the collective responsibility of all participants to uphold these principles in order to maintain an open and supportive atmosphere $[6,7]$.

\section{Structured Meeting Format}

Increasing evidence suggests that a structured and transparent approach to $M \& M$ meeting results in measurable gains in user satisfaction and participation as well as educational benefit, overall patient safety and quality of care $[12,20,33]$. Tools that have been used to guide focussed and goal-directed discussion include the SBAR (Situation, Background, Assessment, Recommendations) model and Vincent, Taylor-Adams and Stanhope's framework for analysing medical error [10, 11, 32, 34]. A structured case presentation format based on the SBAR (Situation, Background, Assessment, Recommendations) framework has been shown to improve the quality of presentations and educational outcomes in the surgical setting [18, 19]. Such a framework encourages systematic analysis of the sequence of events and vulnerabilities in the patient pathway that have contributed to patient harm. An important component of this evaluation is a root cause analysis to identify specific factors contributing to the adverse event. These include factors related to human error, systems error and patient-related causes [7, 18]. Another key element is to present relevant evidence from the literature to guide discussion and identify learning points [9, 19].

\section{Recommendations and Dissemination}

Successful system change requires identification of the problem, formulation of a solution, followed by a robust process for implementation of the agreed action plan [7]. All recommendations should be SMART (Specific, Measurable, Achievable, Realistic, Timely) and assigned to a responsible party with an agreed timeframe for completion $[7,10,11,19]$. The implementation of an action plan may lead to a quality improvement project and a subsequent audit showing progress would close the loop of the patient safety feedback cycle [10-12, 33].

It is important to provide feedback to M\&M participants with updates on the implementation of previously agreed recommendations, which encourages future engagement in 
the M\&M process [10]. Furthermore, communication channels to disseminate key M\&M messages across departments and the wider organisation should be explored to facilitate organisational learning from common and recurring themes $[10,11,35,36]$.

\section{Recommendations for IR M\&M Meetings}

\section{Case identification}

- Each local IR service should agree on a set of criteria for selecting cases for discussion. This will vary based on local practice and case-mix e.g. regional major trauma centres, units offering oncology services.

- Cases may be self-reported by the treating IR team, referred from clinical teams or identified through institutional patient safety reporting systems [10].

- Recommended specific criteria for inclusion are:

Never-events including wrong site procedures

Post-procedural inpatient deaths

Complication requiring additional unplanned post-procedural therapy or prolonged hospital stay.

Complication resulting in permanent sequelae or unplanned readmission within 30 days.

Near misses and incidents reported through local patient safety reporting systems.

- The timing of a complication may be intra-operative, peri-operative or delayed. Late complication is defined in the surgical literature as those observed at least 1-month post-procedure [3, 37].

- Incidents that breach local radiation protection guidelines should be discussed and addressed. These include patient doses that exceed local diagnostic reference levels, trigger compulsory reporting to the regulatory authority, or result in any deterministic effects such as skin erythema.

\section{Meeting frequency and duration}

- M\&M meetings should be held at regular intervals. Meeting frequency depends on the size and workload of each IR unit. For example, once a month is probably adequate for most departments but weekly or fortnightly meetings may be more appropriate for larger and busier centres performing larger volumes of higherrisk cases. As a general rule, shorter and more frequent meetings are preferred [13, 20] as they are more engaging, easier to fit into busy schedules and ensure that the cases are contemporaneous.
- Meetings should be scheduled during normal working hours and ideally during protected time to ensure all relevant staff members are able to attend. They should not coincide with other commitments including IR lists, clinics or diagnostic reporting sessions.

- The time allocated to each case should be proportionate to the severity or complexity of the case, typically ranging from 10 to $30 \mathrm{~min}$. There should be a balance between the case presentation and discussion [7, 32].

\section{Attendees}

- All consultant Interventional Radiologists (IRs) are expected to attend. M\&M meetings should ideally be incorporated into their working timetable to ensure attendance. The presence of senior IRs is important to ensure focussed analysis of events and smooth running of each meeting.

- All IR fellows are also expected to attend and should be encouraged to present the cases at each meeting. Learning from complications is an important part of training and participation in M\&M meetings supports the development of clinically engaged IRs.

- Attendance by the multidisciplinary team involved in IR service delivery should be actively encouraged [6, 10, 13, 20, 32]. These include nurses, radiographers, anaesthetists, surgeons, radiology trainees and medical students. Multidisciplinary participation adds to the depth of the review and organisational memory. All team members who were involved in an adverse event can benefit from meeting discussions [13].

- A record of all attendees should be kept for each meeting. Attendance record may be reviewed for annual appraisal of IR consultants and fellows as evidence of participation in patient safety and quality improvement activity.

\section{Meeting chair}

- Each meeting should be chaired or moderated by a consultant IR. This role requires expert knowledge and leadership experience in order to maintain an open and constructive atmosphere and facilitate focussed discussion among participants [6, 7, 32].

- The meeting chair should ensure that cases identified for discussion meet the agreed departmental criteria, important cases with critical learning points are prioritized, and an agenda for the meeting has been circulated in advance [7].

- The number of cases to be discussed at each meeting should reflect the complexity of cases to be discussed within the fixed allocated meeting time [20]. 
- The meeting chair should maintain a balance between case presentation and discussion and enable the meeting to progress [7].

- The chair should uphold an attitude of non-judgement throughout the meeting, facilitate consensus of opposing views and challenge inappropriate behaviour that hinders the overarching aim of shared learning [6, 7].

\section{Case presentation format}

- A standardized case presentation format should be used to improve efficacy of presentations and maintain consistency in the analysis process, irrespective of the presenter or case. This approach has been shown to improve quality of presentations, enhances focus on relevant details of the case, and facilitates robust analysis of patient care to identify the most salient learning points [18, 19].

- We recommend the SBAR (situation, background, assessment, recommendation) format, which is widely utilised in healthcare and military settings to standardise communication [10, 18, 19, 32]. The following recommended SBAR framework has been modified to reflect IR practice and may be further adapted to meet any specific requirements of each IR unit:

\section{$\mathrm{S}$ (Situation)}

A brief description of the case including basic demographic information, admission diagnosis, procedure undertaken and the adverse outcome.

B (Background)

A summary of relevant clinical information and sequence of events that led to the adverse outcome. These include, existing comorbidities, relevant investigations related to present admission, indication for intervention, outcome of multidisciplinary discussion, any change to the original treatment plan and the rationale for this decision, relevant details of the procedure undertaken, when the complication or adverse event was identified and how it was managed.

A (Assessment and analysis)

A systematic analysis of the sequence of events that led to the adverse outcome and a root cause analysis to identify contributing factors categorised as illustrated in Table 1 [7, 9, 18, 19].

$\mathrm{R}$ (Review of literature \& Recommendations)

A brief summary of relevant literature to promote evidence-based practice and to identify any deviation from established standards. We recommend referring to relevant CIRSE and SIR documents relating to standards of practice and quality improvement. Based on the findings of the root cause analysis and additional knowledge garnered from relevant literature, key learning points are identified with proposed actions to prevent future recurrence.

\section{Discussion and Grading of Complication}

- Once the case has been presented, the meeting chair should open the discussion to the wider group for questions and comments.

- Any questions or clarification regarding the case should be directed to the responsible IR consultant. However, it is preferable for the discussion to be steered mainly by other members of the group [7].

- A consensus should be reached on the key issues that led to the undesired outcome, categorised under one or more headings as outlined in Table 1.

- The adverse outcome should be graded according to the CIRSE Quality Assurance Document on the Classification of Complications [3] as illustrated in Table 2.

- Opposing views or disagreements may require a majority vote. If additional information is deemed necessary, the case may be brought to the next meeting although it is preferable to conclude the case in the same session.

\section{Recommendations and Dissemination}

- Recommendations to address the key issues should be reached by consensus. They need to be concise, proportional to the grade of harm and adhere to the SMART criteria (Specific, Measurable, Achievable, Realistic and Timely) [10, 11, 38]. Each action point should be assigned to a responsible individual or working group with an agreed timeframe for completion [7, 10].

- Key learning points and recommendations from M\&M meetings should be disseminated to all major stakeholders in the IR service including multidisciplinary members of the IR unit, referring clinical groups, and senior management. They should also feed into the wider governance programme of the organisation through incident reporting systems, quality improvement projects and audits [10, 11].

- Any adverse incidents related to medical devices should be reported to the manufacturer and appropriate national agency that regulates the safety and quality of medical devices, such as the Medicines and Healthcare products Regulatory Agency (MHRA) in the UK.

- Sharing important lessons with the wider IR community is encouraged through literature and presentation at scientific meetings, including dedicated M\&M sessions at CIRSE annual congress and the ICCIR meeting. 
Table 1 Categories of contributing factors in root cause analysis

\begin{tabular}{|c|c|}
\hline Category & Examples \\
\hline Human & $\begin{array}{l}\text { Misinterpretation of imaging } \\
\text { Inappropriate case selection } \\
\text { Inadequate experience of primary operator } \\
\text { Deviation from agreed protocols } \\
\text { Delayed recognition of important clinical signs and symptoms } \\
\text { Delay or failure to seek assistance from colleagues }\end{array}$ \\
\hline Technical & $\begin{array}{l}\text { Technical difficulty } \\
\text { Error in judgement } \\
\text { Omission or error during procedure }\end{array}$ \\
\hline System & $\begin{array}{l}\text { Unreliable access to IR suite } \\
\text { Technical issues with imaging equipment } \\
\text { Inadequate staffing levels } \\
\text { Lack of robust on-call arrangements } \\
\text { Poor access to anaesthetic support } \\
\text { Excessive caseload } \\
\text { Poorly coordinated patient pathways } \\
\text { Poor access to inpatient beds } \\
\text { Inadequate handover arrangements }\end{array}$ \\
\hline Education & Deficiency in training and supervision of staff \\
\hline Patient & $\begin{array}{l}\text { Comorbidities } \\
\text { ASA grade } \\
\text { Non-compliance } \\
\text { Refusal to consent to treatment }\end{array}$ \\
\hline Device & $\begin{array}{l}\text { Device malfunction } \\
\text { Incorrect device usage } \\
\text { Device usage outside the scope of IFU (Instructions for Use) }\end{array}$ \\
\hline Medication & $\begin{array}{l}\text { Side-effect } \\
\text { Error in administration } \\
\text { e.g. iv fluids, blood products, antibiotics, sedation, anaesthetic drugs }\end{array}$ \\
\hline
\end{tabular}

Table 2 CIRSE classification system for complications

\begin{tabular}{ll}
\hline Grade & Description \\
\hline 1 & $\begin{array}{c}\text { Complication during the procedure which could not be solved within the same session: no additional therapy, no post-procedure } \\
\text { sequelae, no deviation from the normal post-therapeutic course }\end{array}$ \\
2 & $\begin{array}{l}\text { Prolonged observation including overnight stay (as a deviation from the normal post-therapeutic course }<48 \mathrm{~h} \text { ); no additional post- } \\
\text { procedure therapy, no post-operative sequelae }\end{array}$ \\
3 & $\begin{array}{l}\text { Additional post-procedure therapy or prolonged hospital stay ( }>48 \mathrm{~h} \text { ) required; no post-procedure sequelae } \\
4\end{array}$ \\
$\begin{array}{l}\text { Complication causing permanent mild sequelae (resuming work and independent living) } \\
6\end{array}$ & $\begin{array}{l}\text { Complication causing permanent severe sequelae (requiring ongoing assistance in daily life) } \\
\text { Death }\end{array}$ \\
\hline
\end{tabular}

\section{Feedback}

- The meeting chair should commence all conferences with an acknowledgment of minutes from the previous meeting and an update on the progress of previously agreed recommendations $[10,11]$.
- Recurrent patterns of error should be identified and any barriers to implementation of key recommendations should be escalated to governance leads and senior management [7].

- The meeting chair should escalate any serious breach of conduct by individuals or significant breakdown in 
systems to the governance lead for investigation. If such an investigation is in progress, a summary of the M\&M discussions should be shared with the relevant parties [7].

\section{Administrative Support and Record Keeping}

- M\&M conferences should be supported by a dedicated coordinator who is responsible for keeping a record of attendance, producing meeting minutes and maintaining a database of M\&M cases [7, 9, 13].

- The minutes should contain a summary of the discussions rather than points attributed to individuals. It is important to state clearly the final consensus regarding key root causes, complication grading, and action points assigned to a responsible party.

- Details of individual operators should be removed or anonymised in the meeting minutes and case presentations.

- A copy of the case presentation should be kept with the meeting minutes. This will aid future review and follow-up of recommendations.

- A database of cases will assist with tracking local trends, identifying recurrent issues and monitoring incidence of complications [9].

- All records are confidential and should be treated according to local information governance guidelines.

\section{Conclusions}

As a specialty centred around performing invasive procedures, errors, complications and adverse outcomes are inevitable in delivering an IR service. As our specialty continues to expand, there is an increasing call for IRs to become more clinically engaged and take responsibility for patient care before and after the procedure. M\&M meetings fulfil a dual purpose of learning from error and improving patient safety and are increasingly recognised as an essential component of contemporary medicine. Despite this, there is considerable variation in their structure and limited guidance for best practice, especially in reference to IR. We have outlined a recommended approach to the M\&M conference in this document, based on evidence from other specialties with more experience in the M\&M process. This encompasses an inclusive and blame-free environment and revolves around a continuous cycle of reporting adverse events, structured and goal-directed scrutiny of events, and implementation of SMART actions in a timely manner. Productive M\&M discussions promote a departmental culture that values shared learning and accountability.
Funding This study was not supported by any funding.

\section{Declarations}

Conflict of interest Tiago Bilhim has has speaker agreements with the following companies: Merit Medical, Terumo, Philips, Cook. He is also a stock holder of EmbolX. All other authors declare they have no conflict of interest.

\section{References}

1. Mok PS, Tan EY, Baerlocher MO, Athreya S. The role of morbidity and mortality meetings in interventional radiology. Eur $\mathbf{J}$ Radiol. 2012;81(11):3344-7.

2. Keeling AN, Reekers JA, Lee MJ. The clinical practice of interventional radiology: a European perspective. Cardiovasc Intervent Radiol. 2009;32(3):406-11.

3. Filippiadis DK, Binkert C, Pellerin O, Hoffmann RT, Krajina A, Pereira PL. Cirse quality assurance document and standards for classification of complications: the cirse classification system. Cardiovasc Intervent Radiol. 2017;40(8):1141-6.

4. Dholakia CA, Reavis KM. Morbidity and mortality conference. In: Tichansky DS, Morton JM, Jones DB, editors. The SAGES Manual of Quality, Outcomes and Patient Safety. Boston, MA: Springer; 2012. https://doi.org/10.1007/978-1-4419-7901-8_18.

5. Codman EA. The classic: a study in hospital efficiency: as demonstrated by the case report of first five years of private hospital. Clin Orthop Relat Res. 2013;471(6):1778-83.

6. Orlander JD, Barber TW, Fincke BG. The morbidity and mortality conference: the delicate nature of learning from error. Acad Med. 2002;77(10):1001-6.

7. The Royal College of Surgeons. Morbidity and mortality meetings: a guide to good practice. Royal College of Surgeons; 2015. https://www.rcseng.ac.uk/surgeons/supporting-surgeons/regional/ docs/good-surgical-practice-morbidity-and-mortality-meetings.

8. Liu V. Error in medicine: the role of the morbidity and mortality conference. AMA J Ethics. 2005;7(4):315-9.

9. Tuong B, Shnitzer Z, Pehora C, Choi P, Levine M, Krishnamurthy $\mathrm{G}$, et al. The experience of conducting mortality and morbidity reviews in a pediatric interventional radiology service: a retrospective study. J Vasc Interv Radiol. 2009;20(1):77-86.

10. Giesbrecht V, Au S. Morbidity and mortality conferences: a narrative review of strategies to prioritize quality improvement. Jt Comm J Qual Patient Saf. 2016;42(11):516-27.

11. Fraser J. The morbidity and mortality meeting: time for a different approach? Arch Dis Child. 2016;101(1):4-8.

12. Sinitsky DM, Gowda SB, Dawas K, Fernando BS. Morbidity and mortality meetings to improve patient safety: a survey of 109 consultant surgeons in London. United Kingdom Patient Saf Surg. 2019;13:27.

13. Vreugdenburg TD, Forel D, Marlow N, Maddern GJ, Quinn J, Lander R, et al. Morbidity and mortality meetings: gold, silver or bronze? ANZ J Surg. 2018;88(10):966-74.

14. Antonacci AC, Lam S, Lavarias V, Homel P, Eavey RA. A report card system using error profile analysis and concurrent morbidity and mortality review: surgical outcome analysis. Part II J Surg Res. 2009;153(1):95-104.

15. Heslin MJ, Taylor B, Hawn MT, Davies JE, Heslin RT, Mims $\mathrm{AH}$, et al. A $100 \%$ departmental mortality review improves observed-to-expected mortality ratios and University HealthSystem Consortium rankings. J Am Coll Surg. 2014;218(4):554-62. https://doi.org/10.1016/j.jamcollsurg.2013.12.023.

16. Kennedy I. Learning from Bristol: The Bristol Royal Infirmary Inquiry: The Department of Health's Response to the Report of 
the Public Inquiry into children's heart surgery at Bristol Royal Infirmary 1984-1995. 2002. https://www.gov.uk/government/ publications/the-department-of-healths-response-to-the-report-ofthe-public-inquiry-into-childrens-heart-surgery-at-the-bristol-royalinfirmary.

17. Francis R. Report of the mid staffordshire NHS foundation trust public inquiry. New Directions for Youth Development. 2013. https://gov.uk/government/publications/report-of-the-mid-staffordshirenhs-foundation-trust-public-inquiry.

18. Mitchell EL, Lee DY, Arora S, Kwong KL, Liem TK, Landry GL, et al. SBAR M\&M: a feasible, reliable, and valid tool to assess the quality of, surgical morbidity and mortality conference presentations. Am J Surg. 2012;203(1):26-31.

19. Mitchell EL, Lee DY, Arora S, Kenney-Moore P, Liem TK, Landry GJ, et al. Improving the quality of the surgical morbidity and mortality conference: a prospective intervention study. Acad Med. 2013;88(6):824-30.

20. Higginson J, Walters R, Fulop N. Mortality and morbidity meetings: an untapped resource for improving the governance of patient safety? BMJ Qual Saf. 2012;21(7):576-85.

21. Dagli MS, Soulen MC, McGinn C, Mondschein JI, Clark TWI, Sudheendra D, et al. Impact of a monthly compliance review on interventional radiology adverse event reporting. J Am Coll Radiol. 2019;16(1):73-8.

22. De Vos MS, Hamming JF, Marang-Van De Mheen PJ. Barriers and facilitators to learn and improve through morbidity and mortality conferences: A qualitative study. BMJ Open. 2017; 7(11):018833.

23. Savader SJ, Venbrux AC, Savader BL, Fishman EK, Trerotola SO, Lund GB, et al. Complications of interventional radiology: an imaging overview. Clin Imaging. 1993;17(4):282-91.

24. Parvizi N, Robertson I, McWilliams RG. Medical device adverse incident reporting in interventional radiology. Clin Radiol. 2014; 69(3):263-7.

25. Howe E. Poly Implant Prosthese (PIP) silicone breast implants review of the actions of the MHRA and Department of Health. 2012. https://assets.publishing.service.gov.uk/government/uploads/ system/uploads/attachment_data/file/216537/dh_134043.pdf.

26. European Parlament, Council of the European Union. Regulation (EU) 2017/745 of the European Parliament and of the Council of 5 April 2017 on medical devices. Off J Eur Union. 2017. https:// www.eur-lex.europa.eu/legal-content/EN/TXT/PDF/?uri=CELEX: 32017R0745.
27. Roberts AC. The 2004 Dr. Charles T. Dotter lecture: interventional radiology today - What would Charles Dotter say? J Vasc Interv Radiol. 2004;15(12):1357-61.

28. Murphy TP. Introduction to clinical interventional radiology. Semin Interv Radiol. 2005;22(1):3-5.

29. Lee MJ, Fanelli F, Haage P, Hausegger K, Van Lienden KP. Patient safety in interventional radiology: a CIRSE IR checklist. Cardiovasc Intervent Radiol. 2012;35(2):244-6.

30. Haynes AB, Weiser TG, Berry WR, Lipsitz SR, Breizat A-HS, Dellinger EP, et al. A surgical safety checklist to reduce morbidity and mortality in a global population. $\mathrm{N}$ Engl $\mathrm{J}$ Med. 2009;360(5):491-9.

31. Leoni CJ, Potter JE, Rosen MP, Brophy DP, Lang EV. Classifying complications of interventional procedures: a survey of practicing radiologists. J Vasc Interv Radiol. 2001;12(1):55-9.

32. Joseph CW, Garrubba ML, Melder AM. Informing best practice for conducting morbidity and mortality reviews: a literature review. Aust Heal Rev. 2018;42(3):248-57.

33. Pang DSJ, Rousseau-Blass F, Pang JM. Morbidity and mortality conferences: a mini review and illustrated application in veterinary medicine. Front Vet Sci. 2018;5:43.

34. Vincent C, Taylor-Adams S, Stanhope N. Framework for analysing risk and safety in clinical medicine. BMJ. 1998;316(7138): 1154-7.

35. Dargon PT, Mitchell EL, Sevdalis N. Morbiditty and mortality conference manual v.1.1 Imperial College London and Oregon Health and Science University. 2012. https://imperial.ac.uk/ media/imperial-college/medicine/surgery-cancer/pstrc/mmmanualv1. 1dec2012rev.pdf.

36. Bechtold ML, Scott S, Nelson K, Cox KR, Dellsperger KC, Hall LW. Educational quality improvement report: outcomes from a revised morbidity and mortality format that emphasised patient safety. Qual Saf Health Care. 2007;16(6):422-7.

37. Dindo D, Demartines N, Clavien PA. Classification of surgical complications: a new proposal with evaluation in a cohort of 6336 patients and results of a survey. Ann Surg. 2004;240(2): 205-13.

38. Bowman J, Mogensen L, Marsland E, Lannin N. The development, content validity and inter-rater reliability of the SMARTgoal evaluation method: a standardised method for evaluating clinical goals. Aust Occup Ther J. 2015;62(6):420-7.

Publisher's Note Springer Nature remains neutral with regard to jurisdictional claims in published maps and institutional affiliations. 